\title{
Synthesis and Characterization of Nano Size Conducting Polyaniline
}

\author{
CH. Srinivas ${ }^{1}$, D. Srinivasu ${ }^{2}$, B. Kavitha ${ }^{3}$, N. Narsimlu ${ }^{4}$, K. Siva Kumar ${ }^{5}$ \\ 1,2,3,4,5 Department of Physics, Osmania University, Hyderabad 500 007, INDIA
}

\begin{abstract}
Conducting polymers have attracted considerable attention in the recent years due to their unique thermal, electrical, optical and magnetic properties. These polymers have been conducive for fabrication of micro-electronic devices. Among these polymers, Polyaniline (PANI) possesses an exceptional properties including structural, thermal and electrical. We have synthesized PANI in Emeraldine salt form by chemical oxidation method with controlled pH3 by using Ammonium perdisulphate as oxidizing agent. The morphology of PANI has been determined by using X-Ray Diffraction (XRD), Scanning Electron Micrograms (SEM) and Dynamic Light Scattering (DLS). The studies revealed that Polyaniline possess rod like shape with radius around $75 \mathrm{~nm}$ and length around $617.6 \mathrm{~nm}$. The DSC thermogram shows two exothermal peaks at around 97.07 ${ }^{\circ} \mathrm{C}$ and $152{ }^{\circ} \mathrm{C}$, these peaks are attributed to the glass transition temperature (Tg) and PANI degradation temperature respectively. The final valley point (endothermic) around $175{ }^{\circ} \mathrm{C}$ is attributed to the melting point $\left(T_{m}\right)$ of PANI. The voltage-current (V-I) characteristics of Polyaniline have studied at temperature $300 \mathrm{~K}$. Due to semi crystalline solid nature of PANI, the transport of charge carriers may takes place in between and along PANI chains.
\end{abstract}

Key Words: Polyaniline, XRD, DLS, DSC, V-I characteristics, Exponent ' $m$ '

\section{Introduction}

Conjugated Polymers unravel a new field of condensed matter physics called as Plastic electronics and draw a wide attention. Since the discovery of conjugated polymers, a tremendous amount of research has been carried out in the field of conducting polymers. The fabrication of polymer diode (in 1980) and light emitting diode (in 1990) boosted up the field of conducting polymer ${ }^{1,2}$. The unique electronic properties of conjugated organic polymers are owing to conjugated $\Pi$-electrons present on the back bone of polymer. The conductivity of conjugated polymers may be tuned by several orders of magnitude just as in inorganic semi-conductors. This tunable conductivity can be achieved by various doping mechanisms like 'chemical doping by charge transfer', 'electrochemical doping', 'photo doping', 'charge induction at metal semiconductor interface' and 'doping by acid-base chemistry'2.

Among the conducting polymers, Polyaniline is an excellent example of П-conjugated polymer. Polyaniline can be synthesized for specific applications like rechargeable batteries, biosensors, corrosion protection materials, organic electronic materials. The conductivity and I-V characteristics of Polyaniline depend on different experimental conditions like temperature, $\mathrm{pH}$ value, concentration of oxidant and on the nature of do-pant. Among different methods of synthesize of Polyaniline, the chemical oxidation and electrochemical synthesis are prominent ${ }^{3}$. The chemical oxidation polymerization is practically viable due to feasibility of synthesis mechanism for producing large scale Polyaniline powder. The inception of Nitrogen heteroatom in between Phenyl rings of Polyaniline backbone chain results the significant properties of PANI ${ }^{4}$. Generally Polyaniline exists in three forms namely Leucoemaraldine, Permigraniline and Emaraldine. In the Emaraldine form, it exists in insulating state [Emaraldine base (EB)] and conducting state [Emaraldine salt $(E S)]$ with conductivity $(\sigma)$ around $10^{-10} \mathrm{~S} / \mathrm{cm}$ and around $10^{1} \mathrm{~S} / \mathrm{cm}$ respectively. Emaraldine salt form is obtained upon protonation of Emaraldine Base with protonic acids i.e., oxidative doping. The fundamental properties of polyaniline depend on the mechanism of charge transfer in the polymer ${ }^{5}$. The presence of localized electronic states and formation of polarons and bipolarans in the energy band led to the possibility of different types charge conduction. In this paper, the synthesis and characterization of nanosized Polyaniline with different experimental techniques have been discussed. The characteristics can surface the applicability of the synthesized PANI for fabrication of Rectifier and Photodiode.

\section{Experimental Section}

Synthesis

The chemical oxidative polymerization of aniline has been carried out in aqueous solution at $0^{0} \mathrm{C}$ by dissolving aniline in a strong acidic solution $1 \mathrm{M} \mathrm{HCl}$. The polymerization is initiated by drop wise adding of aqueous Ammonium perdisulphate solution. The reaction is highly exothermic, hence the addition rate of oxidant is properly controlled. At the time of polymerization $\mathrm{pH}$ of solution should be controlled to 3 to get 
emaraldine form of polyaniline. After complete addition of oxidant, this mixture is stirred for $10 \mathrm{hrs}$ with a Teflon coated magnetic stirrer on ice bath for complete polymerization. After polymerization, the deep green mixture is filtered by gravity filtration method and washed with doubly ionized $\mathrm{H}_{2} \mathrm{O}$ and dilute $\mathrm{HCl}$ until the filtrate become colorless. Further addition of protonic acid, i.e., $\mathrm{HCl}$ doping, convert the Emaraldine base form into Emaraldine salt form. Finally the Polyaniline has been sintered in a carbide rod furnace at $60^{\circ} \mathrm{C}$ for 5 hrs to get Polyaniline powder.

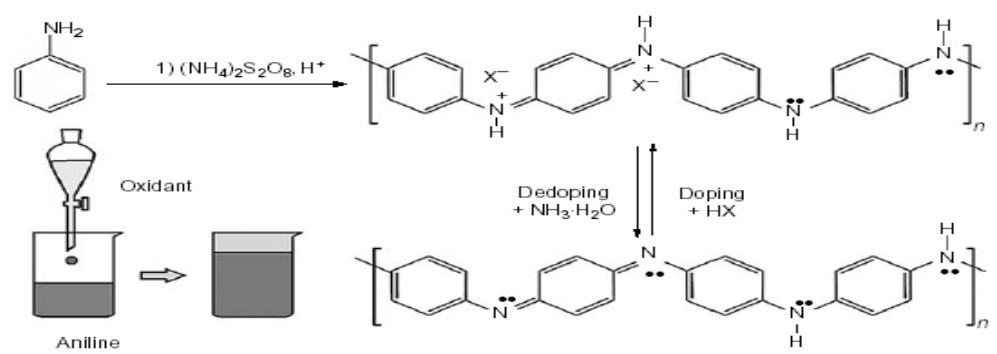

Fig.1.Polymerization mechanism of aniline monomer

\subsection{Characterization}

The synthesized Polyaniline powder is pelletized with help of hydraulic machine at 5 Tons pressure. These pellets are used to study the morphology of polyaniline with X-Ray diffraction (XRD). The XRD pattern is recorded with help of Phillips XPERT diffractometer by using $\mathrm{Cu}-\mathrm{K} \alpha$ X-rays of wave length $1.54 \AA$. Scanning Electron Micrograms are recorded with help of Hitachi SEM experiment in Forevision Technologies. The Dynamic Light Scattering experiment has been carried to study size distribution analysis of polyaniline by dispersion in doubly ionized $\mathrm{H}_{2} \mathrm{O}$ by employing the Malvern DLS equipment. The results have been obtained at count rate of $146.7 \mathrm{kcps}$ with duration of use as $70 \mathrm{sec}$, measurement position $5.50 \mathrm{~mm}$ and attenuator as 10 . The corresponding results are plotted as Size of sample particles vs \%Intensity scattered by the particles. The Differential Scanning Calorimetric (DSC) studies are performed on Perkin Elmer model DSC7, USA from $50^{\circ} \mathrm{C}$ to $300^{\circ} \mathrm{C}$ at a scan rate of $20^{\circ} \mathrm{C} / \mathrm{min}$ heat flow. The Current-Voltage (I-V) characteristics are studied at room temperature by using Keithley 614 electrometer.

\section{Results And Discussion}

The Fig. 2 shows X-ray diffraction of Polyaniline recorded at room temperature with two diffraction peaks at $2 \theta=21.10^{\circ}$ and $23.59^{\circ}$ respectively.

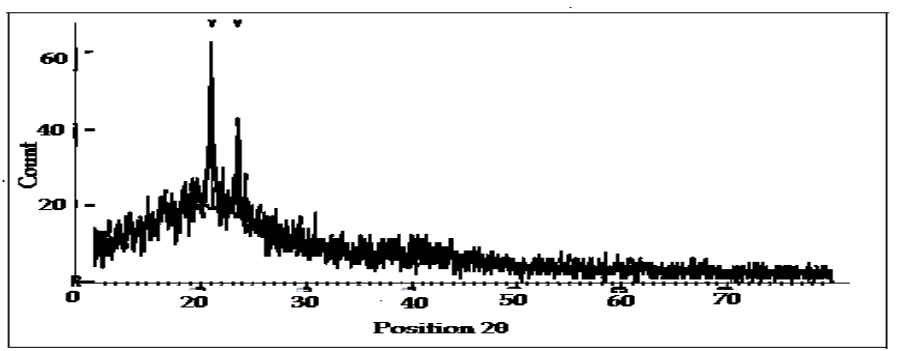

Fig. 2 XRD pattern of Polyaniline Emaraldine salt

The pattern shows sharp and well defined peaks, which indicate semi-crystalline nature of Polyaniline. The plains of Benzinoid and Quinoid rings of PANI chain are responsible for crystalline structure. The interplanar distance and crystallite size are estimated by Bragg's law and Debye Scherer equation $\{\mathrm{D}=[0.89 \lambda] /[\beta \operatorname{Cos} \theta]\}$ respectively. Where $\beta$ is a full width half maxima $(\mathrm{FWHM})^{6,7}$.

Table-1 Physical Parameters of Polyaniline from XRD Studies

\begin{tabular}{|c|c|c|c|}
\hline $\operatorname{Cos}(2 \theta)$ & FWHM & d-spacing & Crystallite size \\
\hline 21.1007 & 0.1574 & $42.1047 \mathrm{~nm}$ & $8.021 \mathrm{~nm}$ \\
\hline 23.5995 & 0.3149 & $37.7001 \mathrm{~nm}$ & $9.331 \mathrm{~nm}$ \\
\hline
\end{tabular}

The SEM image of Polyaniline clearly indicates that the polymer possess nano-fiber like structure with length around $600 \mathrm{~nm}$ (Fig.3). This image also reveals that PANI contains some pores or voids. The Dynamic Light Scattering studies on Polyaniline have been conducted in water medium at room temperature $\left(25^{\circ} \mathrm{C}\right)$. The results posses two size distribution peaks at around $150.1 \mathrm{~nm}$ (radius around $75 \mathrm{~nm}$ ) and $617.6 \mathrm{~nm}$ 
(Fig.4). These two peaks are assigned to the diameter and lengths of PANI particles. The sizes of PANI particles measured in SEM and DLS are almost equal, but in XRD the crystallite sizes are very less. These results indicate that PANI is a cluster of some crystallites.

The Differential Scanning Calorimetric (DSC) graph of PANI is recorded from $50{ }^{\circ} \mathrm{C}$ to $300{ }^{\circ} \mathrm{C}$ at $20{ }^{\circ} \mathrm{C}$ per minute heat flow. The thermograph shows two exothermal peaks at $97.07{ }^{\circ} \mathrm{C}$ and at around $152^{\circ} \mathrm{C}$ (Fig. 5). These peaks are attributed to the Glass transition temperature ( $\mathrm{Tg}$ ) and PANI degradation temperature respectively. The final valley point (endothermic) around $175{ }^{\circ} \mathrm{C}$ is attributed to the melting point $\left(\mathrm{T}_{\mathrm{m}}\right)$ of PANI ${ }^{6}$.

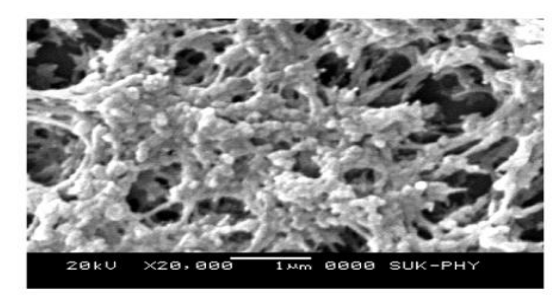

Fig. 3 SEM image of Polyaniline

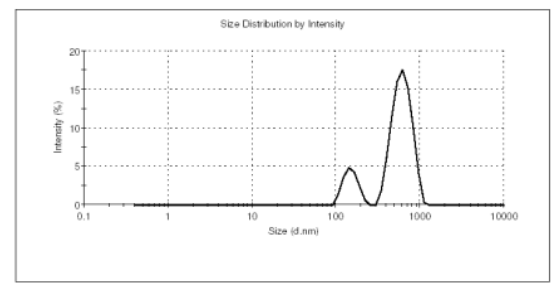

Fig. 4. DLS report of Polyaniline

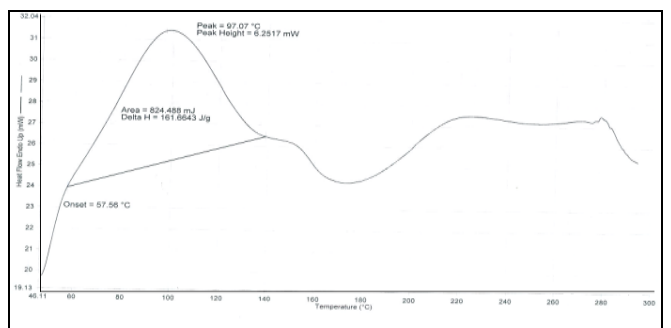

Fig. 5 DSC thermograph of Polyaniline (heat flow from $50{ }^{\circ} \mathrm{C}$ to $300{ }^{\circ} \mathrm{C}$ at $20{ }^{\circ} \mathrm{C}$ per min.)

The current-voltage (I-V) characteristic of Polyaniline exhibits non-linear nature for Emaraldine salt (Fig.6). In PANI, polarans and bipolarans are produced due to doping of protonic acid. The non- ohmic behavior reveals that the contribution of charge transport is due to polarans and bipolarans in addition to free charge carriers (electrons and holes) ${ }^{8,9}$. The relation between $\mathrm{V}$ and I may be expressed as

$$
\mathrm{I}=\mathrm{A} \mathrm{V}^{\mathrm{m}}
$$

where ' $\mathrm{A}$ ' is a constant and ' $\mathrm{m}$ ' is the exponent which is determined from slope of the $\mathrm{I}-\mathrm{V}$ characteristic curve.
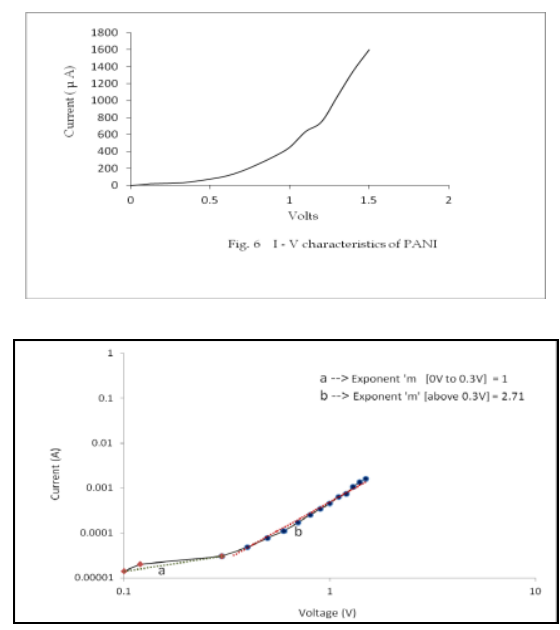

Fig. 7 I - V curve of PANI plotted on a log-log scale

www.iosrjournals.org 
The I-V characteristics of PANI on Log-Log scale has been shown in Fig.7. It is evident that, there exist a transition between two linear regions of the curve at 0.3 Volts. Fig.7 reveals that, the I-V characteristics between $0 \mathrm{~V}$ to $0.3 \mathrm{~V}$ has been linear and the corresponding exponent ' $\mathrm{m}$ ' for this region is around 1 . Thus PANI behaves as ohmic material within the region of $0 \mathrm{~V}$ to $0.3 \mathrm{~V}$.

The second region of I-V characteristics above 0.3 Volts has an exponent ' $m$ ' around 2.71. Thus, PANI has non-ohmic in nature. It clearly indicates that Pure PANI has non-ohmic in nature above 0.3 volts. This property is greatly useful in the fabrication of rectifiers and photodiodes.

\section{Conclusions}

Polyaniline nano-fibers have been synthesized by chemical oxidative method. The morphological studies (XRD, SEM and DLS) indicate the radius of polymer fiber is around $75 \mathrm{~nm}$ and length around $617.6 \mathrm{~nm}$. The DSC thermogram of PANI shows two exothermal peaks $\left(97.07{ }^{0} \mathrm{C}, 152{ }^{\circ} \mathrm{C}\right.$ ), which are assigned to thermal glass transition temperature $\left(\mathrm{T}_{\mathrm{g}}\right)$ and Polymer degradation Temperature. An Endothermic peak is existing at $175{ }^{0} \mathrm{C}$, which indicates melting point of Polyaniline. The I-V characteristics of PANI indicate that charge transport is due to mobility of polarans and bipolarans apart from electrons and holes, in between PANI chains. The I-V characteristic curve of PANI is clearly indicating the non-ohmic nature above 0.3 Volts of applied potential.

\section{Acknowledgments}

The authors are thankful to the Head Department of Physics, Osmania University, Hyderabad, India, for providing laboratory facilities.

\section{REFERENCES}

[1] Jianyong Ouyang, Chih-Wei Chu, Ricky J.Seng, Ankitha Prakash and yang Yang. Hand Book of conducting polymers.Processing and applications.8.-1Third Edition (2006)

Heeger A.J , Nobel lecture December 8 (2000)

Gupta K, Jana P.C, and Melkap, A.K, Journal of Physical Sciences. 12, 233-238. (2008)

Narsimlu N, Kuo Y.D, Chun Guey Wu Journal of applied polymer science 88, 3183-3187, (2003)

Ghosh M, Mekap A.K, Chattopadhyay, Chatterjee. Journal of Physics and chemistry of Solids 62, 475-484 (2001)

Cheng-Ho Chen. Journal of Applied Polymer science. 89, 2142-2148 (2003)

Gupta K.K, Jasnal M, Agarwal K, Indian Journal of fiber and Textile research33, 443-450 (2008)

[8] Jiaxing Huang and Richar B.Kaner, Hand Book of conducting Polymers.Processing and

Applications. 7-3, Third Edition (2006)

[9] Wang, Lee W.T, Reed M.A Phys. Rev. B.68-035416. 2003 